\title{
O uso da metformina na gravidez: uma revisão integrativa da literatura
}

\author{
The use of metformin in pregnancy: an integrative literature review \\ El uso de metformina en el embarazo: una revisión bibliográfica integradora \\ Carlos Enrique Campos Asenjo ${ }^{1 *}$, Luis Alberto Leon Camac.
}

\begin{abstract}
RESUMO
Objetivo: Revisar sobre os traços científicos disponível sobre a utilização e o uso da metformina no período de gravidez. Métodos: Foi realizado o método de revisão bibliográfica, desenvolvida com a seleção de artigos em base de dados de pesquisas em saúde Scielo, Lilacs, Medline e PubMed, utilizando-se dos seguintes descritores: Metformina; Gravidez; Diabetes gestacional. Resultados: Não existe relatos nas literaturas de aumento a complicações através do consumo da metformina como forma de tratar a diabetes durante a gestação. Os efeitos colaterais que podem vir surgir são sugestivos aos gastrointestinais, náuseas e diarréia, mas que diminuem, com a redução da dose ou ingestão alimentar concomitante. Referente a sua eficácia no controle glicêmico foi verificado que a metformina e a insulina impetram resultados compatíveis, com a glicemia em jejum a ser similar nos dois grupos. Aconselha-se que os fatores de risco para o DMG sejam investigados na primeira consulta do pré-natal. Considerações finais: A metformina demonstraser um medicamento seguro durante o período gestacional, não evidenciando aumento de taxas de malformações congênitas, sepsis neonatal, morte do feto ou traumas durante o parto,diante das buscas de base cientifica.Salienta-se que a metformina é um fármaco que consegue desempenhar função no tratamento do diabetes. Seu uso no período gestacionalpermite a diminuição deocorrência e complicações referente à doença, como a diabetes gestacional, o risco de pré-eclâmpsia e de aborto.
\end{abstract}

Palavras-chaves: Metformina, Gravidez, Diabetes mellitus gestacional.

\begin{abstract}
Objective: Review available scientific traits on the use and use of metformin during pregnancy. Methods: A bibliographic review method was developed, based on the selection of articles from the Scielo, Lilacs, Medline and PubMed health research database, using the following descriptors: Metformin ; Pregnancy; Gestational diabetes. Results: There are no reports in the literature of increased complications from the use of metformin as a treatment for gestational diabetes. Some side effects that appear are gastrointestinal, such as nausea and diarrhea, but over time decrease with dose reduction or concomitant food intake. In terms of efficacy in glycemic control, metformin and insulin were found to have comparable results, with fasting glucose being similar in both groups. Risk factors for GDM are recommended to be investigated at the first prenatal visit. Conclusion: Metformin has been shown to be safe during pregnancy, with no evidence of increased rate of congenital malformations, neonatal sepsis, fetal death or trauma. during childbirth by literature. It is noteworthy that metformin is a drug that plays an important role in the treatment of diabetes. Its use throughout pregnancy reduces the incidence of some complications associated with the disease, such as gestational diabetes, preeclampsia and risk of miscarriage.
\end{abstract}

Keywords: Metformin, Pregnancy, Gestational diabetes mellitus.

\section{RESUMEN}

Objetivo: Revisar los rasgos científicos disponibles sobre el uso y el uso de metformina durante el embarazo. Métodos: Se desarrolló un método de revisión bibliográfica, basado en la selección de artículos de la base de datos de investigación de salud Scielo, Lilacs, Medline y PubMed, utilizando los siguientes descriptores:

1 Universidade Federal do Amazonas (UFAM), Manaus-AM. *E-mail: ceca.02@hotmail.com 
Metformina; Embarazo Diabetes gestacional. Resultados: No hay informes en la literatura sobre el aumento de complicaciones por el uso de metformina como tratamiento para la diabetes gestacional. Algunos efectos secundarios que aparecen son gastrointestinales, como náuseas y diarrea, pero con el tiempo disminuyen con la reducción de la dosis o ingesta de alimentos concomitante. En términos de eficacia en el control glucémico, se encontró que la metformina y la insulina tienen resultados comparables, siendo la glucosa en ayunas similar en ambos grupos. Se recomienda investigar los factores de riesgo para la DMG en la primera visita prenatal. Conclusión: Se ha demostrado que la metformina es segura durante el embarazo, sin evidencia de un aumento en la tasa de malformaciones congénitas, sepsis neonatal, muerte fetal o trauma. durante el parto por la literatura. Es notable que la metformina es un medicamento que juega un papel importante en el tratamiento de la diabetes. Su uso durante el embarazo reduce la incidencia de algunas complicaciones asociadas con la enfermedad, como diabetes gestacional, preeclampsia y riesgo de aborto espontáneo.

Palabras clave: Metformina, Embarazo, Diabetes mellitus gestacional.

\section{INTRODUÇÃO}

A gravidez é acompanhada por resistência à insulina, mediada principalmente pela secreção placentária de hormônios diabetogênicos, incluindo hormônio de crescimento, hormônio liberador de corticotropina, lactogênio placentário (somatomammotropina coriônica), prolactina e progesterona. Essas e outras alterações metabólicas garantem que o feto tenha uma ampla oferta de nutrientes (DURNWALD CMD, 2018).

A resistência à insulina se dá no começo do segundo trimestre e aumenta durante todo o período gestacional. Os hormônios placentários são grandes responsáveis pela resistência à insulina, pois modificam os mecanismos fisiológicos imprescindíveis, que garantem todoo aporte glicêmico para o feto, mudando o metabolismo dos macronutrientes quando relacionado a uma resposta energética (MONTENEGROCAB; REZENDE FILHO J, 2008).

A Diabetes mellitus gestacional (DMG) é caracterizada pela condição de intolerância a carboidratos, o que implica em hiperglicemia de agravamento variável, com início ou diagnóstico durante a gestação. Sua fisiopatologia é elucidada pelo aumento de hormônios contra-reguladores da insulina, devido ao estresse fisiológico atribuído pela gravidez e a fatores acondicionados por condições genéticas ou ambientais.

O hormônio que está diretamente conexo com a resistência à insulina durante o período gestacional é o hormônio lactógeno placentário, contudo, é evidente que já exista outros hormônios hiperglicemiantes como cortisol, estrógeno, progesterona e a prolactina (SOCIEDADE BRASILEIRA DE ENDOCRINOLOGIA E METABOLOGIA, 2006).

O diabetes é uma síndrome que pode ser caracterizada como a condição de distúrbios metabólicos heterogêneos assinalados por hiperglicemia cujo resultado ocasiona defeitos na secreção e ação da insulina, ou ambos (ASSOCIAÇÃO AMERICANA DE DIABETES, 2014).

Diabetes mellitus gestacional (DMG) é a intolerância aos carboidratos diagnosticada pela primeira vez durante a gestação e que pode ou não persistir após o parto (OPPERMANN MLR et al, 2004). É um problema metabólico comumente na gestação tendo predomínio de $3 \%$ e $25 \%$ das gestações, o que ode diversificar por meio do grupo étnico, da população e do critério diagnóstico escolhido (SACKS DA, 2012). Podendodesenvolver o diabetes mellitus tipo 2 (DM2) no período gestacional.

A incidência da DMG teve um acréscimo nos últimos anos, devido à alteração dos critérios de diagnóstico que, baseados pelo estudo Hapo SCR (2008), a incidência de DMG teve umvalor anormal quando analisado a glicemia, abrangendo uma porcentagem significante de grávidas antecipadamente classificadas como saudáveis (AMERICAN DIABETES ASSOCIATION, 2015).

O diabetes gestacional é uma das causas que mais atinge o metabólico, sendo um problema comumente diagnosticado na gestação e que tem predominio de $3 \%$ e $25 \%$ das gestações, tendo variações quando correlacionado a grupo étnico, da população e de qual será a forma de conduta do tratamento que será utilizado (SCHMIDT MI, et al, 2001 e SACKS DA, 2012). 
A investigação de DMG deve ser realizada em todas as gestantes sem diagnóstico prévio de diabetes. Entre a vigésima quarta $\left(24^{a}\right)$ e a vigésima oitava $\left(28^{a}\right)$ semana de gestação; deve-se realizar o teste oral de tolerância à glicose TOTG com dieta sem restrição de carboidratos ou com, no mínimo, ingestão de $150 \mathrm{~g}$ (grama) de carboidratos nos três dias anteriores ao teste, com jejum de oito horas (SBD, 2015).

$O$ uso de antidiabéticos orais durante o período de gravidez, como a metformina, vem sendo bastante ingerido, devido à existência de fatores clínicos favoráveis. (AMERICAN DIABETES ASSOCIATION,2015).

Em âmbito global, os antidiabéticos orais podem ser separados em: os que aumentam a secreção pancreática de insulina; e as que retardam a velocidade de absorção de glicídios, tem os que enfraquecem a produção hepática de glicose e os que acrescentam a utilização periférica de glicose (SOCIEDADE BRASILEIRA DE DIABETES, 2014)

A metformina é qualificada pela US FoodandDrugAdministration, (PEIXOTO CILS, 2016), como fármaco e sua classificação está direcionada em categoria $B$, aquelas que não apresenta efeito teratogénicos nos animais.

Exceções de carboseenquadram na mesma categoria, sendo uma vez que outros antidiabéticos orais foram classificados como categoria $\mathrm{C}$, desconhecendo os riscos fetais que poderá surgir (AMERICAN DIABETES ASSOCIATION,2015).

Como fármaco de primeira escolha para tratamento dediabetes mellitus tipo 2 (DM2), a metformina vem sendo o anti-hiperglicemiante oral mais prescrito, devido a apresentação relevantedo aspecto de toxicidade 0 tornando benfeitorquanto a sua eficácia clínica.

Da visão geral clínica a metformina é um fármaco que agrega interessante no perfil terapêutico, pois tem grande valor ao ser utilizada como agente antidiabético conseguindo estender a outras situações patológicas, dentre elas: síndrome metabólica, síndrome do ovário policístico (SOP), hirsutismo e também utilizada como regulador do ciclo menstrual (DOWLING RJO, et al., 2011).

A metformina consegue reduzir alguns níveis de glicose, o que resulta na diminuição da gliconeogênese hepática, ajustando um declínio médio refente a níveis de insulina, promovendo a absorção de glicose no músculo (ALEXANDER GC, et al., 2008).

O cloridrato de metformina deriva da guanidina, é composto por ativo da Galega officinalis. Planta medicinal, que tem como sinonímia popular Lilac francês, de utilização por séculos na Europa como tratamento do diabetes desde a época medieval (GRAHAM GG, et al., 2011).

Administrada por via oral, absorvida de forma incompleta e lenta pela parte superior do intestino delgado, o que retarda sua absorção, mas que não é prejudicada por presença de alimentos na bolsa estomacal. No epitélio intestinal, a metformina é absorvida na borda em escova e é um importante substrato da proteína PMAT (plasma membranemonoaminetransporter).

Os transportadores dos cátions orgânicos 1(OCT1) e transportadores dos cátions orgânicos 2(OCT2), envolve-se com a entrada da metformina no fígado e rins, respectivamente (TAKANE H, et al., 2008)

Embora não existam relatados de efeitos fetais contrário em relação a sua utilização, a metformina atravessar a placenta e com isso conseguedanificar a fisiologia fetal, sedo assim a sua utilização durante 0 período gestacionalcontestável (ROWAN JA,2008). Sendo assim o presente trabalho tem como objetivo revisar na literaturasobre os traços científicos disponíveis sobre a utilização e o uso da metformina no período de gravidez.

\section{MÉTODOS}

A pesquisa se faz por meio de revisão bibliográfica, utilizando como fonte de dados à literatura científica existente sobre metfomirna na gravidez. As fontes para a seleção das publicações foram bases de dados eletrônicas sendo estes: Scientific Eletronic Library online (SCIELO) e Literatura Latino-americana e do Caribe em Ciências da Saúde (LILACS), Medical Literature Analysis and Retrievel System Online (Medline) e US National Library of Medicine (PubMed). 
A busca dos dados foi realizada de acordo com os seguintes descritores: Metformina; Gravidez; Diabetes gestacional. Utilizando os pressupostos contidos em meio aos critérios, de inclusão e exclusão. Foram utilizados como critérios de inclusão artigosde 2010 a 2019 na língua portuguesa, espanhola e inglesa, com o tema relacionado a metformina na gravidez e diabetes mellitus, e a partir dos descritores citados, foram encontrados 30 artigos relacionados ao tema, e para os critérios de exclusão: desconsiderou artigos que não proporcionavam o tema na íntegra, sendo 11 selecionados buscando o objetivo do trabalho (Figura 1).

Figura 1 - Estabelecimento dos critérios de inclusão e exclusão dos artigos nas bases de dadosutilzadas: Scientific Eletronic Library online (SCIELO) e Literatura Latino-americana e do Caribe em Ciências da Saúde (LILACS) Medline e PubMed.
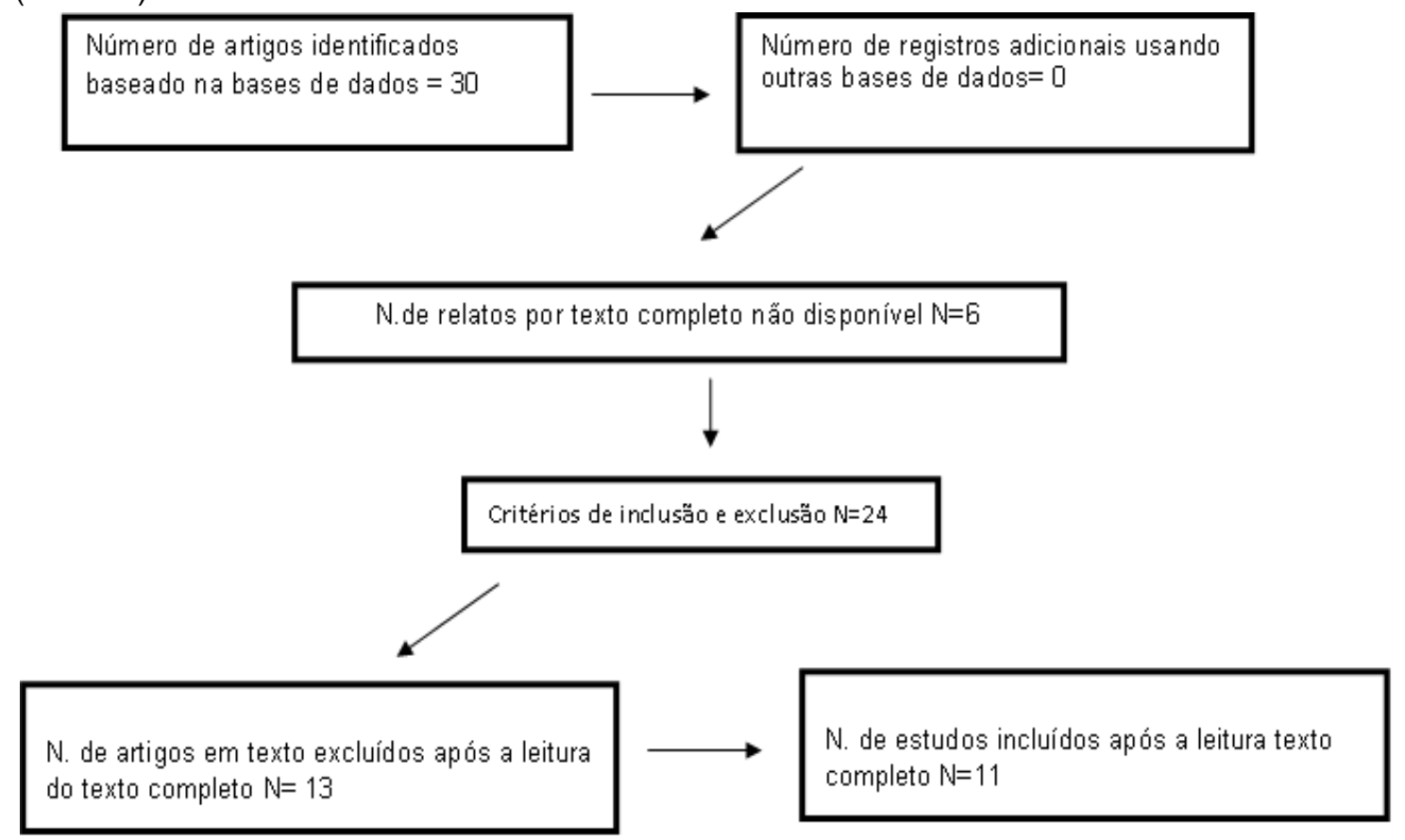

Fonte: Asenjo CEC, et al., 2019.

\section{RESULTADO E DISCUSSÃO}

Em estudos já concluídos sobre a utilização da metformina ainda não foi comprovadoalgum aumento considerável em função de complicações quanto a utilização da metformina no tratamento da diabetes gestacional. Langer, O (2010), afirma que estes efeitos colaterais existentes são de origem gastrointestinal, náuseas e a diarréia, que se restringem ao longo do tempo de uso. Já um dos efeitos considerado lateral grave é a acidose láctica, que tem maior incidência e ocorre raramente em doentes com função e hepática normais.

O mesmo é compartilhado por Milech A e Oliveira JEP, (2004), onde ele reafirma que um dos efeitos contrários maior queixa ao uso da metformina é a intolerância gastrointestinal, que chega a ser em média de $20 \%$ dos pacientes. Relatos apontam também o gosto metálico, anorexia, náuseas, distensão abdominal e diarréia, os quais, geralmente, são autolimitados e tendem a se resolver com a continuação do tratamento.

Estratégias para minimizaroacontecimento destes efeitos é a utilização do consumo do medicamento juntamente com refeições comacréscimo da dose gradual, a cada 7 dias, sempre estando dentro dos os níveis glicêmicos afim de conseguir atingir as metas terapêuticas. Em contrapartida cerca de $5 \%$ dos pacientes são integralmente intolerantes ao fármaco, assim como foram descritos no Quadro 1. 
Quadro 1 - Distribuição das referências por autor, ano, título e resultados encontrados.

\begin{tabular}{|c|c|c|}
\hline Autor/ano & Título do trabalho & Resultados \\
\hline $\begin{array}{c}\text { Kovo } \\
\text { M,(2008) }\end{array}$ & $\begin{array}{l}\text { Determinação da transferência } \\
\text { de metformina através da } \\
\text { placenta humana usando um } \\
\text { modelo de cotilédone } \\
\text { placentário ex vivo com dupla } \\
\quad \text { perfusão }\end{array}$ & $\begin{array}{l}\text { Seis instalações experimentais completas foram concluídas. As taxas de transporte materno-fetal para } \\
\text { metformina e antipirina foram } 10,61 \pm 2,85 \% \text { e } 30,98 \pm 5,62 \% \text {, respectivamente. O índice de depuração, } \\
\text { calculado como a razão entre as permeabilidades da metformina e antipirina, foi de } 0,34 \pm 0,05 \text {. }\end{array}$ \\
\hline $\begin{array}{c}\text { Rowan } \\
\text { JA,(2015) }\end{array}$ & $\begin{array}{c}\text { Metformina versus insulina no } \\
\text { tratamento da diabetes } \\
\text { gestacional }\end{array}$ & $\begin{array}{c}\text { Das } 363 \text { mulheres designadas para metformina, } 92,6 \% \text { continuaram recebendo metformina até o parto e } 46,3 \% \\
\text { receberam insulina suplementar. A taxa do desfecho composto primário foi de } 32,0 \% \text { no grupo designado à } \\
\text { metformina e } 32,2 \% \text { no grupo da insulina (risco relativo, 1,00; intervalo de confiança de } 95 \%, 0,90 \text { a 1,10). Mais } \\
\text { mulheres no grupo da metformina do que no grupo da insulina declararam que escolheriam receber o tratamento } \\
\text { designado novamente }(76,6 \% \text { vs. } 27,2 \%, P<0,001) .\end{array}$ \\
\hline $\begin{array}{l}\text { Tertti K, et } \\
\text { al, (2014) }\end{array}$ & $\begin{array}{l}\text { O grau de exposição fetal à } \\
\text { metformina não influencia o } \\
\text { resultado fetal no diabetes } \\
\text { mellitus gestacional }\end{array}$ & $\begin{array}{l}\text { Duzentos e dezessete pacientes com DMG foram randomizados para metformina. Concentrações foram } \\
\text { determinadas por espectrometria de massa no soro materno às } 36 \text { semanas gestacionais (gw) e ao nascimento, } \\
\text { e no sangue do cordão umbilical. A proporção média de concentração de metformina no cordão umbilical / soro } \\
\text { materno foi de } 0,73 \text {. Não houve diferenças no peso ao nascer entre pacientes tratados com insulina e } \\
\text { metformina estratificados por tercis mínimos de concentração de metformina medidos em } 36 \text { gw. A taxa de } \\
\text { hipoglicemia neonatal }(p=0,92) \text { e o valor de } p H \text { da artéria umbilical }(p=0,78) \text { foi semelhante nos pacientes } \\
\text { tratados com insulina e metformina, estratificados por tercis de concentração de metformina no cordão umbilical. }\end{array}$ \\
\hline $\begin{array}{l}\text { Waheed S, } \\
\text { et al, } \\
\text { (2013) }\end{array}$ & $\begin{array}{l}\text { Eficácia da metformina versus } \\
\text { insulina no tratamento da } \\
\text { gravidez com diabetes. }\end{array}$ & $\begin{array}{c}\text { A idade média foi de } 29,82 \pm 4,58 \text { e } 29,35 \pm 4,97 \text { anos nos grupos A e B, respectivamente. Açúcar no sangue } \\
\text { em jejum o nível após } 1 \text { mês foi controlado em } 22(64,7 \%) \text { pacientes do grupo A e em } 27(79,4 \%) \text { do grupo B } \\
\text { (p> 0,05). Sangue em jejum nível de açúcar no termo, permaneceu controlado em } 30 \text { (88,2\%) pacientes do } \\
\text { grupo A e } 27(79,4 \%) \text { no grupo B }(p>0,05) \text {.Comparação dos níveis aleatórios de açúcar no sangue dentro dos } \\
\text { limites normais após } 1 \text { mês em } 25(73,5 \%) \text { no grupo A e em } 24(70,6 \%) \text { no grupo B. No termo, o nível aleatório } \\
\text { de açúcar no sangue foi controlado em } 28(82,4 \%) \text { e } 27(79,4 \%) \text { pacientes do grupo A e B,respectivamente.A } \\
\text { eficácia da metformina e insulina no controle do diabetes foi igual em dois grupos. }\end{array}$ \\
\hline
\end{tabular}

REAS/EJCH | Vol.Sup.n.42 | e2082 | DOI: https://doi.org/10.25248/reas.e2082.2020 Página 5 de 10 


\begin{tabular}{|c|c|c|}
\hline $\begin{array}{l}\text { Bertoldo } \\
\text { MJ et al, } \\
\text { (2014) }\end{array}$ & $\begin{array}{c}\text { Impacto da metformina nos } \\
\text { tecidos reprodutivos: uma } \\
\text { visão geral da gametogênese } \\
\text { à gestação }\end{array}$ & $\begin{array}{c}\text { Foi demonstrado que esta droga ativa uma proteína quinase denominada proteína quinase ativada por AMP } 5 \\
\text { 'ou AMPK. AMPK está presente em muitos tecidos, tornando o efeito da metformina multifatorial. No entanto, } \\
\text { como a metformina atravessa a placenta, seu uso durante a gravidez suscita preocupações em relação a } \\
\text { possíveis efeitos adversos na mãe e no feto. A maioria dos relatórios não sugere efeitos adversos significativos } \\
\text { ou teratogenicidade. }\end{array}$ \\
\hline $\begin{array}{l}\text { Sivalingam } \\
\text { VN, et al } \\
(2014)\end{array}$ & $\begin{array}{l}\text { Metformina em saúde } \\
\text { reprodutiva, gravidez e câncer } \\
\text { ginecológico: indicações } \\
\text { estabelecidas e emergentes }\end{array}$ & $\begin{array}{l}\text { Entre as } 4.977 \text { mulheres estudadas, } 2,4 \% \text { (IC95\% } 2,0-2,9) \text { apresentaram os critérios GDMby ADA e } 7,2 \% \text { (6,5- } \\
\text { 7,9) pelos critérios da OMS. Da mesma forma, a GDM, segundo os critérios da OMS, previa aumento do risco de } \\
\text { macrossomia }(1,45,1,06-1,95) \text {, pré-eclâmpsia }(1,94,1,22-3,03) \text { e morte perinatal }(1,59,0,86-2,90) \text {. Das } \\
\text { mulheres positivas pelos critérios da OMS, } 260 \text { (73\%) foram negativas pelos critérios da ADA. Por outro lado, } 22 \\
(18 \%) \text { mulheres positivas pelos critérios da ADA foram negativas pelos critérios da OMS }\end{array}$ \\
\hline $\begin{array}{c}\text { Waheed S, } \\
\text { (2013) }\end{array}$ & $\begin{array}{l}\text { Eficácia da metformina versus } \\
\text { insulina no tratamento da } \\
\text { gravidez com diabetes }\end{array}$ & $\begin{array}{c}\text { A idade média foi de } 29,82 \pm 4,58 \text { e } 29,35 \pm 4,97 \text { anos nos grupos A e B, respectivamente. Açúcar no sangue } \\
\text { em jejum o nível após } 1 \text { mês foi controlado em } 22(64,7 \%) \text { pacientes do grupo A e em } 27(79,4 \%) \text { do grupo B } \\
\text { (p> 0,05). A comparação do nível de HBA1C pós-tratamento mostra que o diabetes controlado em } 27(79,4 \%) \\
\text { pacientes do grupo A enquanto em } 28 \text { (82,3\%) pacientes do grupo B. A eficácia da metformina e insulina no } \\
\text { controle do diabetes foi igual em dois grupos. }\end{array}$ \\
\hline $\begin{array}{l}\text { Amirl FN, } \\
\text { et al., } 2018\end{array}$ & $\begin{array}{c}\text { Fatores de Risco para } \\
\text { Gestacional Diabetes Mellitus: } \\
\text { A Case-Control Study }\end{array}$ & $\begin{array}{l}\text { Depois de se ajustar as variáveis, a análise multivariada identificou } 4 \text { factores de risco significativos da DMG, } \\
\text { incluindo pré-gestacional baixa actividade física (odds ratio [OR] intervalo de confiança }=2,85,95 \%[\mathrm{Cl}]=0,97- \\
\text { 8,34), a idade materna avançada (OR }=1,24,95 \mathrm{Cl} \%=1,13-1,35) \text {, índice de massa corporal } \cdot 30 \mathrm{~kg} / \mathrm{m} 2(\mathrm{OU} \\
=1,10, \mathrm{IC} 95 \%=1,02-1,20) \text {, e histórico familiar de diabetes mellitus (OR }=5,62, \mathrm{IC} \text { de } 95 \%=2,26-13,96) \text {. } \\
\text { Observou-se associações significativas entre a baixa actividade física pré-gestacional e obesidade com risco } \\
\text { DMG. Assim, a conclusão deste estudo pode ajudar a estratégias de conceber para a prevenção da GDM. }\end{array}$ \\
\hline
\end{tabular}

REAS/EJCH | Vol.Sup.n.42 | e2082 | DOI: https://doi.org/10.25248/reas.e2082.2020 Página 6 de 10 


\begin{tabular}{|c|c|c|}
\hline $\begin{array}{l}\text { Peixoto } \\
\text { CILS, et al., } \\
(2016)\end{array}$ & $\begin{array}{l}\text { A utilização da metformina } \\
\text { durante a gravidez }\end{array}$ & $\begin{array}{l}\text { A metformina exerce efeitos através do aumento da sensibilidade à insulina e da diminuição da produção } \\
\text { hepática e absorção intestinal de glicose, o que em teoria é benéfico para a preservaçáo das células } \\
\text { pancreáticas- } \beta \text { 7. Seus efeitos são exercidos pela inibição do complexo I da cadeia respiratória mitocondrial, } \\
\text { levando a maior produção de ATP e, consequentemente, de AMP. Este ativa a proteína-cinase ativada por AMP } \\
\text { (AMPK), que está frequentemente inativada na obesidade e insulinorresistência. }\end{array}$ \\
\hline $\begin{array}{l}\text { Pinheiro C } \\
(2008)\end{array}$ & $\begin{array}{l}\text { A metformina é eficaz na } \\
\text { diabetes gestacional }\end{array}$ & $\begin{array}{l}\text { O resultado primário, resultante da compilação das complicações neonatais anteriormente descritas, não revelou } \\
\text { diferença estatisticamente significativa entre o grupo da metformina e o grupo da insulina ( } 32 \% \text { versus } 32,2 \% \text {; } \\
\text { IC:0,8-1,23; } p=0,95) \text {. A hipoglicemia grave foi menos frequente no grupo da metformina }(3,3 \%, 8,1 \% ; P=0,08) \text {, } \\
\text { mas o parto prematuro (antes da } 37^{a} \text { semana de gestação) foi mais frequente neste grupo }(12,1 \% \text { versus } 7,6 \% \text {; } \\
\text { IC: } 1,02-2,52 ; p=0,04) \text {. (LOE=1b-) }\end{array}$ \\
\hline $\begin{array}{l}\text { San Martin- } \\
\text { Herrasti } \\
\text { JMS (2014) }\end{array}$ & $\begin{array}{c}\text { A metformina e diabetes } \\
\text { gestacional. }\end{array}$ & $\begin{array}{l}\text { Constatou-se que o uso de metformina é aceito } 76,6 \% \text { dos pacientes e foi eficaz em } 53,7 \% \text { dos casos; quando } \\
\text { adicionado era menos necessidades de insulina e as complicações quando utilizado somente insulina. A } \\
\text { prevalência de complicações ou efeitos colaterais foi baixa pelo que a sua utilização durante a gravidez é } \\
\text { seguro. }\end{array}$ \\
\hline $\begin{array}{l}\text { AlizaH, et } \\
\text { al., (2017) }\end{array}$ & $\begin{array}{l}\text { Metformina versus insulina } \\
\text { Tratamento em diabetes } \\
\text { gestacional na gravidez e os } \\
\text { seus efeitos no peso ao } \\
\quad \text { nascer Neonatal }\end{array}$ & $\begin{array}{l}\text { A idade gestacional média dos pacientes no momento do arrolamento no estudo foi de } 28.13 \pm 2,30 \text { semanas no } \\
\text { grupo tratado com metformina e } 28,26 \pm 2,46 \text { semanas no grupo insulina ( } p \text {-valor } 0,82 \text { ). Ganho de peso médio } \\
\text { foi de } 8,96 \pm 1,78 \mathrm{~kg} \text { em mães que tomam terapia de metformina e } 9,36 \pm 1,40 \mathrm{~kg} \text { em terapia tomar insulina mãe } \\
\text { (valor de } p 0,33 \text { ). A taxa de cesariana foi de } 43,3 \% \text { no grupo tratado com metformina e } 36,7 \% \text { no grupo da } \\
\text { insulina com um valor } p \text { de } 0,59 \text {. No grupo tratado com metformina, a média de peso dos bebés na altura do } \\
\text { nascimento foi de } 3,2 \pm 0,20 \mathrm{~kg} \text { contra } 3,67 \pm 0,19 \mathrm{~kg} \text { no grupo de insulina }(p<0,001 \text { ). }\end{array}$ \\
\hline
\end{tabular}

Fonte: Asenjo CEC, et al., 2019.

REAS/EJCH | Vol.Sup.n.42 | e2082 | DOI: https://doi.org/10.25248/reas.e2082.2020 Página 7 de 10 
A transferência da metformina chega a ser de 10 a $16 \%$ materno-fetal, como já concluído por estudos realizados ex vivo, colocando em razão a fisiologia fetal e o desenvolvimento do embrião. (KOVO M,2008). Rowan JA (2015), em seu estudo deensaio clínico randomizado obtido com 751 grávidas, verificou que a metformina não etavava ligada ao aumento dos índices das taxas de malformações congénitas, sepse neonatal, morte fetal, trauma no momento do parto ou pré-eclâmpsia.

Como relatado por Um Jamal et al, a utilização prolongada de metformina durante toda a gravidez (em mulheres com síndrome do ovário policístico) reduz as complicações obstétricas sem risco de prova de malformações. Estão em curso esforços para a sua co-administração com a insulina para tratar a diabetes tipo 1.

Já em 2013, o estudo prospectivo randomizado com 221 grávidas, buscou a avaliar a transferência placentária in vivo, concluindo o achado de forma que a exposição fetal à metformina passou a ter significância de aproximadamente dos 73\%(TERTTI K, et al, 2014). Transferência esta que evidenciaser livre do grau de exposição materna.

De acordo com estudos concluídos a metformina desempenha seus efeitos aumentando a sensibilidade à insulina e reduzindo a produção hepática e absorção intestinal de glicose, o que teoriacamente é benéfico afim de preservar as células pancreáticas (WAHEED S, et al, 2013).

Em outro estudo Bertoldo MJ et al, (2014) relata que os efeitos da metformina são completados por inibição do complexo I da cadeia respiratória mitocondrial, aumentando a produção de ATP e, conseguinte, de AMP.Namesma vertente Sivalingam VN, et al (2014), ainda conclui que seus efeitos ativam a proteínacinaseestá ativada por AMP (AMPK), que está constantemente inativada na obesidade e insulinoresistência.

Estudos deWaheed S, (2013), relata que a metformina consegue diminuir níveis glicémicos sem trazerriscos de hipoglicemia ou ocasionar um aumento ponderal, vantajoso por ser fácil de administrar, além de ter baixo custo associado. (RAI $L$ et al,2009). Em relação a eficácia no controle glicêmico, estudos feitos por ljäs $\mathrm{H}$ et al, (2011) e Niromanesh S, et al, (2012) verificou-se que a metformina e a insulina obtêm resultados comparáveis, com a glicemia em jejum sendo assim semelhantes nos dois grupos.

O rastreamento precoce também é fortemente recomendado para que se identifique o diabete prévio à gestação, mas ainda não diagnosticado, atualmente definido como overt diabetes (AMERICAN DIABETES ASSOCIATION 2011).

Um ensaio prospectivo randomizado desenvolvido por KhattabS, et al, (2011), com 257 mulheres com SOP que foram tratadas com metformina no início do primeiro trimestre, não obteveníveis de reduçãopara pré-eclâmpsia, diabetes gestacional e parto pré-termo. Já em um estudo de coorte prospectivo concretizado com 360 grávidas, houve redução significativa, em relaçãoa incidência de diabetes gestacional, e quando utilizado a metformina durante todo o tempo de gestação. (KHATTAB $S$ et al, 2011).

A American Diabetes Association (ADA) de acordo com MetzgerBE, (2002), aconselha que os fatores de risco parao DMG sejam investigados na primeira consulta de pré-natal. De acordo com esta recomendação, seria considerado risco para DMG: a idade igual ou superior a 25 anos; o peso materno excessivo; a história familiar de diabete em parentes de primeiro grau; as alterações prévias do metabolismo de glicose; histórico obstétrico relacionados ao diabete e etnia de risco para diabetes.

A hiperglicemia materna acompanha-se de maior risco de pré-eclâmpsia, hipertensão gestacional e desenvolvimento de diabetes mellitus tipo 2 depois da gravidez (BEN-HAROUSH A,2004). Já Abolfazl MHT et al., (2008), existe riscos relacionados ao aumentado de morte fetal, parto pré-termo, hiperbilirrubinemia, hipoglicemia neonatal, síndrome de dificuldade respiratória e macrossomia.

No estudo de Pinheiro C, (2008), a análise incluiu 363 grávidas inicialmente tratadas com metformina e 370 insulinotratadas. Um número de gestantes foi selecionado para o grupo da metformina, sendo que $46,3 \%$ necessitaram de complementar a terapêutica com insulina e 27 interromperam o tratamento com metformina antes do parto.

Nesse caso o tratamento com metformina foi iniciado com dosagem de $500 \mathrm{mg} / \mathrm{dia}$ em alguns casos feito uso por duas vezes/dia com comida e destinado até uma dose máxima de $2.500 \mathrm{mg} / \mathrm{d}$. Quando esse controle 
glicémico não foi atingido agregou-se o uso da insulina. A metformina é um antidiabético oral eficaz, frequentemente usado na diabetes tipo 2(SIVALINGAM VN, et al,2014).

Outro fator desfavorável associado à metformina é a deficiência de vitamina B12, em tratamentos de longos períodos. Correlação está que vem sendo estudada há algum tempo, contudo, o mecanismo que leva a esta deficiência ainda não foi totalmente esclarecido (TING RZ, 2006).

A eficácia de metformina por si só para os doentes de controle com diabetes mellitus gestacional varia $53,7-90 \%$. É descrito que entre 10 e $46,3 \%$ dos doentes necessitaram de metformina tratadas para o controlo de insulina metabólica (PEIXOTO CILS et al., 2016).

Doentes tratados com metformina são mais propensos a ter parto prematuro (antes das 37 semanas de gestação) em comparação com aqueles que utilizam a insulina sozinha, mas nenhuma diferença em recémnascidos sofre unidade de socorro ou entrada respiratória cuidados intensivos neonatais por este motivo (SUA DF, et al., 2014)

É valido ressaltar que embora até este período não existam relatos de efeitos adversos, é imprescindível acautela na indicação rotineira destas medicações a mulheres com diabetes pré-gestacional. Vários estudos têm demonstrado a segurança da metformina, pode ser considerada como a droga de primeira escolha para iniciar o tratamento de pacientes diagnosticados com diabetes mellitus gestacional (MARQUES P, et al., 2014).

\section{CONSIDERAÇÕES FINAIS}

A metformina tem mostrado efeitos benéficos durante o período gestacional, não tem contribuído parao aumentode taxa de malformações congénitas, sepsis neonatal, ou morte fetal durante os partos. É um fármaco que cumpre importante papel no tratamento do diabetes. Quanto correlacionado ao tratamento da diabetes gestacional é eficazse comparado à da insulina, e mais utilizado pelas mulheres. Em casos da síndrome do ovário policístico, a metformina traz benefícios ao tratamento prévio e em técnicas de reprodução assistida, sendo assim alcança melhor índice em taxas de gravidez, buscano a prevenção da síndrome de hiperestimulação ovárica. Em caso de uso contínuo ao longo do período gestacional consegue diminuir caso de possíveis complicações coligadas à doença, como a diabetes gestacional, pré-eclâmpsia e risco de aborto.

\section{REFERÊNCIAS}

1. ALEXANDER GC, et al. National trends in treatmentof type 2 diabetes mellitus, 1994-2007. Arch Intern Med, v. 168, p. 2088-2094, 2008.

2. AMERICAN DIABETES ASSOCIATION - ADA. Diagnosis and classification of diabetes mellitus. Diabetes Care, v. 37, n. 1, p. 81-90, 2014.

3. AMERICAN DIABETES ASSOCIATION- ADA. Diagnosis and classification of diabetes mellitus. Diabetes Care 2011 Jan;34 Suppl 1: S62-9.

4. AMERICAN DIABETES ASSOCIATION. Standards of Medical Care In Diabetes-2015. Diabetes Care 2015;38 Suppl 1: S8-S79.

5. BERTOLDO MJ, et al. Impact of metformin on reproductive tissues: an overview from gametogenesis to gestation. Ann TransI Med 2014; 2:55.

6. DOWLIN RJO et al. Understanding the benefit of metformin use in cancer treatment. BMC Medicine, v. 9, n. 3, 2011.

7. DSBD- Diretrizes Sociedade Brasileira de Diabetes $2014-2015$

8. DURNWALD, CMD. Diabetes mellitus gestacional: controleglicêmico e prognósticomaterno. 2018.https://www.uptodate.com/contents/gestational-diabetes-mellitus-glycemic-control-and-maternalprognosis/contributors.

9. GRAHAM GG. et al. Clinical pharmacokinetics of metformin. Clinical pharmacyokinetics, v. 50, n. 2, p. 81-98, 2011

10. HAPO SCR. Hyperglycemia and adverse pregnancy outcomes. N Engl J Med 2008; 358:1991-2002

11. IJÄS H, et al. Metformin should be considered in the treatment of gestational diabetes: a prospective randomised study. BJOG 2011; 118:880-885.

12. KHATTAB S, et al. Can metformin reduce the incidence of gestational diabetes mellitusin pregnant women with polycystic ovary syndrome? Prospective cohort study. Gynecol Endocrinol 2011;27(10):789-793.

13. KOVO M, et al. Determination of metformin transfer across the human placenta using a dually perfused ex vivo placental cotyledon model. Eur J ObstetGynecolReprod Biol 2008; 136:29-33 
14. LANGER O. When diet fails: insulin and oral hypoglycemicagents as alternatives for the management ofgestational diabetes mellitus. J Matern Fetal Neonatal Med 2002; 11:218-225.

15. METZGER BE, et al. Hyperglycemia and adverse pregnancy outcomes. N Engl J Med 2008; 358:1991- 2002.

16. MILECH, A; OLIVEIRA, JEP. Diabetes mellitus clínica, diagnóstico, tratamento multidisciplinar. São Paulo: Atheneu, 2004.

17. MONTENEGRO CAB; REZENDE FILHO J. Obstetrícia Fundamental. 11. ed. Rio de Janeiro: Guanabara Koogan, 2008.

18. NIROMANESH S. Metformin compared with insulin in the management of gestational diabetes mellitus: a randomized clinical trial. Diabetes Res Clin Pract 2012; 98:422-429.

19. OPPERMANN MLR. Diabetes e gestação. In: Duncan BB, Schmidt MI, Giuglia-ni ERJ (eds.). Medicina ambulatorial: condutas de atenção primária baseadas em evidências. 3. ed. Porto Alegre: Artes Médicas, 2004; p. 376-82

20. RAI L, et al Metformin a convenient alternative to insulin for Indian women with diabetes in pregnancy. Indian $\mathrm{J}$ Med Sci 2009; 63:491-497.

21. ROWAN JA, et al. Metformin versus insulin for the treatment of gestational diabetes. N Engl J Med 2008; 358:20032015.

22. SACKS DA, et al. Frequency of gestational diabetes mellitus at collaborating centers based on IADPSG consensus panel-recommended criteria: The Hyperglycemia and Adverse Pregnancy Outcome (HAPO) Study. Diabetes Care. 2012 Mar;35(3):526-8

23. SCHMIDT ML, et al. Gestational diabetes mellitus diagnosed with a 2-h 75-g oral glucose tolerance test and adverse pregnancy out comes. Diabetes Care. 2001 Jul;24(7):1151-5

24. SIVALINGAM VN, et al. Metformin in reproductive health, pregnancy and gynaecological cancer: established and emerging indications. Hum Reprod Update 2014;20(6):853-868.

25. SOCIEDADE BRASILEIRA DE DIABETES. Medicamentos orais no tratamento do diabetes mellitus: como selecionálos de acordo com as características clínicas dos pacientes.2014-15

26. TAKANE H, et al. Polymorphism in human organic cation transporters and metformin action. Pharmacogenomics, v. 9, n. 1, p. 415- 422, 2008.

27. TERTTI K, et al. The degree of fetal metformin exposure does not influence fetal outcome in gestational diabetes mellitus. Acta Diabetol 2014;51(5):731-738.

28. TING RZ. Risk factor sof vitamin B12 deficiency in patients receiveing metformin. Archives Internal Medicine, v. 166, n. 18, 2006.1975-1979.

29. WAHEED $S$, et al. Efficacy of metformin versus insulin in the management of pregnancy with diabetes. $J$ Coll Physicians Surg Pak 2013;23(12):866-869. 